\title{
An overview of nearly a half century of microembolic signal processing techniques.
}

\author{
Jean-Marc GIRAUlt ${ }^{1,2}$, Maroun Geryes ${ }^{3}$, Jamal Charara ${ }^{3}$, Sébastien MÉnigot ${ }^{1,2}$ \\ ${ }^{1}$ UMR CNRS 6613 LAUM, LE MANS \\ ${ }^{2}$ Groupe ESEO , 10 boulevard Jeanneteau, 49107 ANGERS, \\ ${ }^{3}$ Department of Physics and Electronics, Faculty of Sciences I, Lebanese University \\ jean-marc.girault@eseo.fr, sebastien.menigot@eseo.fr, maroungeryes@gmail.com
}

\begin{abstract}
Micro-emboli detection for patients with high risk of strokes has been performed with transcranial Doppler (TCD) systems since 1969. As a consequence, instrumentation of TCD systems progressed with the introduction of multigate systems, power mode systems and robotized probes, to name but a few. These new types of TCD have increase the chance of robust detections of quite big micro-emboli and at the same time increased the efficiency of artefact rejection.

For a couple of years now, it is now possible to prevent cerebrovascular accidents (CVA) by detecting very small micro-emboli, the latter being precursor signs of strokes. For this sake, a new generation of transcranial Doppler (TCD) systems (holter) is used to record examinations of long duration. In an attempt to detect the smallest possible micro-emboli, offline softwares based on recent signal processing techniques complete advantageously these holter systems.

In this communication, an overview of fifty years of research developments in embolic signal processing is proposed. What is interesting during this adventure of a half century is that detection methods were inspired as signal processing discoveries coming from speech processing to econometric. With the advent of the artificial intelligence, new challenges are being drawn up.
\end{abstract}

Development of devices dedicated to evaluate the cerebral haemodynamic and the detection of microembolic signatures dates back to 1969. Merill Spencer in the US and Rune Aaslide in Europe, were pioneers in such technologic adventure. They were involved in the development of the first Doppler systems for medical applications. David H. Evans from Leicester and Hugh S. Markus from Cambridge were certainly the two english researchers who contributed the most to the field of emboli characterisation and detection. They wrote more than one hundred papers in that field, two of them that are interesting could be the two following [1,2].

To enable the captation of Doppler signals coming from cerebral arteries, settings of such ultrasound transcranial Doppler systems were adapted to cross the skull. The first detection techniques were based on listening to the ultrasound Doppler signals while they are audibles. For an examination of one hour, a physician had to listen and count the passage of microembolic signatures. The time-consuming and boring tasks were not completely perfect because of the well-known temporal and frequency masking effects making undetectable audio files [3].

To overcome these drawbacks automatic counting of microembolic signals were implemented in commercial devices such as TransCranial Doppler (TCD) systems (see Figure 1). To improve the robustness and sensitivity detection of micro-embolic signatures, several instrumental innovations were integrated into commercial TCD such as multigates [4], multifrequencies [5], the power m-mode Doppler [6], robotized probes [7] , to name

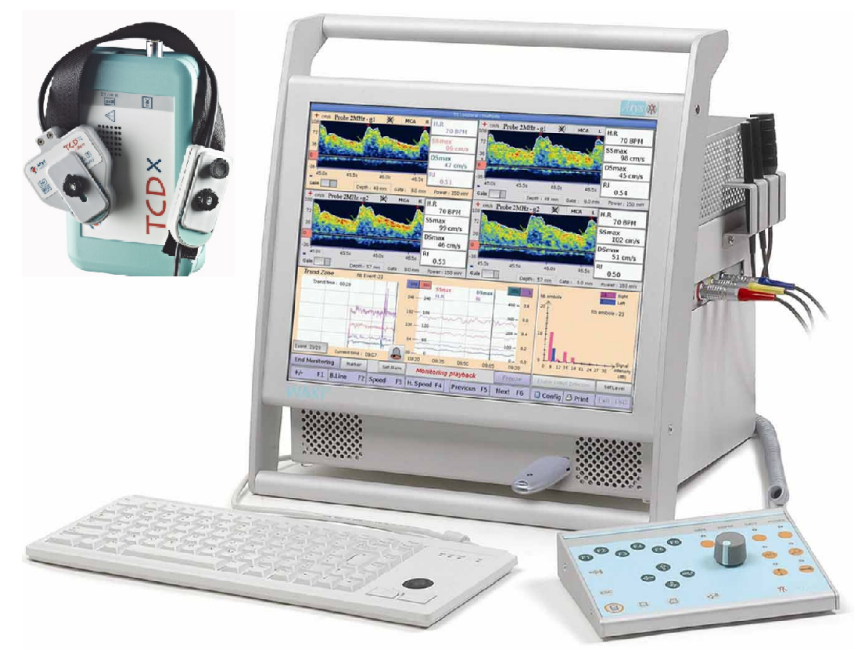

FIGURE 1 - Portable TransCranial Doppler (TCD) system and a TCD holter from Atys Medical.

but a few.

The outline of the proposed document will be guided by the properties of the microembolic signal to be detected. The sounds produced by the microemboli being very different from the circulating blood because of their localized nature in time and frequency, a first group of methods was based on the search for time-frequency characteristics. By taking inspiration from the methods of speech compression, a new group of approaches 


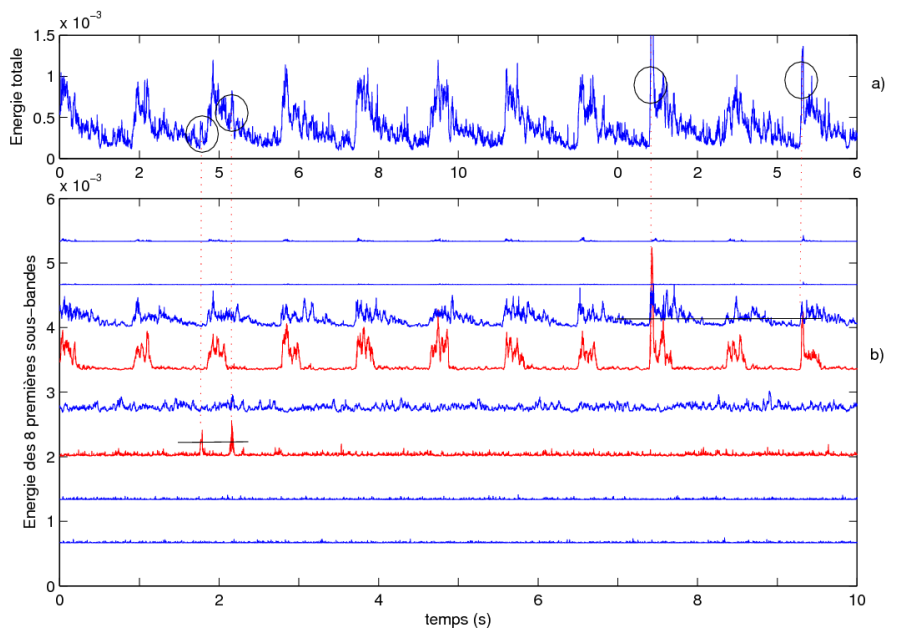

FIGURE 2 - Energy signals obtained through a slidding window from the whole band Doppler signal (a) and from subband Doppler signals (b). Two over-intensities were detected in the subband 3 and two others in the subband 5 while only two overintensities are detected from the whole band Doppler signal.

based on the signals synthesis from models emerged. On the other hand, to take account of the fact that the micro-embolic signal is neither cyclostationary nor heteroskedastic, a new type of methods was proposed. Finally, to mimic the behavior of clinicians during the decision process, a final group of methods based on machine learning were proposed.

Most of micro-emboli detection methods were focused on searching for transient over-intensity signatures in the Doppler signal. Usually this search was done by calculating an energy information through the extraction of few milliseconds of the Doppler signal. Such an estimation could be done directly in the time-domain or in the frequency domain. The micro-embolic signature, being both localized in the Doppler frequency and in the time domains, gave birth to the use of time-frequency or time-scale methods.

The simplest method to be implemented was the short-time Fourier transform (STFT) also called the spectrogram. Today, the spectrogram for 10 seconds Doppler signal is displayed on the screen of portable TCD systems. The own limitations of the STFT led to investigate new time-frequency representations such as the Wigner Ville distribution and its variants [8]. Time-scale representations based on wavelet transforms were also investigated $[9,10,11,12,13,14]$. To optimize timefrequency detection of micro-emboli, matching-pursuit detectors [15] were also tested.

Instead of using time-frequency or time-scale transformations, filter bank systems can replace advantageously such transformations in a real time point of view. Such an approach based on the use of a bank of juxtaposed filters is equivalent to dividing the spectral band into several narrow sub-bands. The detection can be operated independently in each subband/channel. Depending on the spectral division, several filter types can be found : bank of narrow band filters with the same width [16],

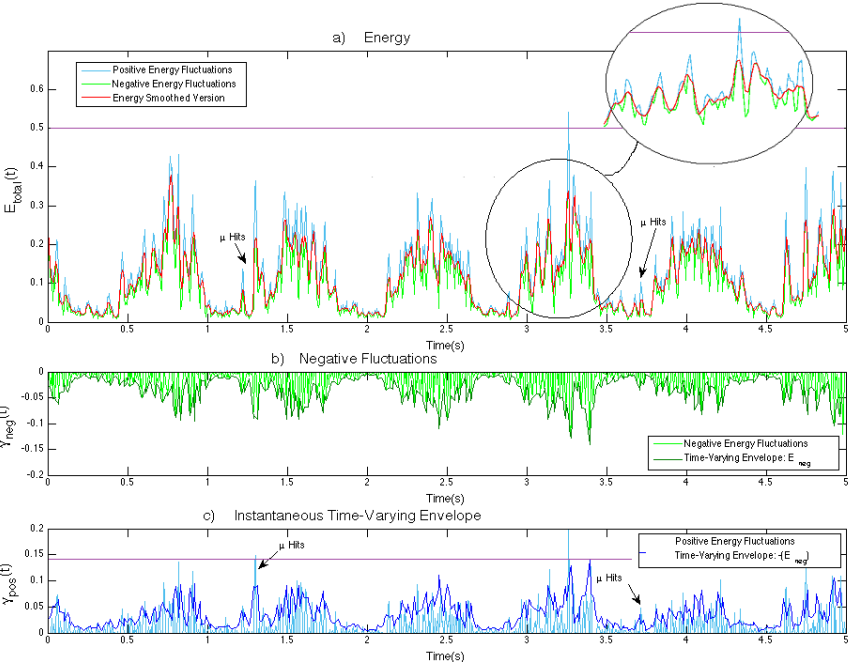

FIGURE 3 - Time-varying energy derived from a Doppler signal and local trend (a). Envelop and negative fluctuation energy (b). This envelop serves as a threshold for the positive energy fluctuation (c).

discrete wavelet decomposition [17], and wavelet packet decomposition [14]. By using these different kinds of filters, the difficulty lies in the choice of the constant threshold in each sub-band and on the fusion of the detection since a microevent can appear in several consecutive sub-bands. Time varying energy calculated from each channel are reported in Figure 2.

Most previous methods were focused on the event detection from energy signals. Another way is to detect rapid change in the Doppler signal model. In a theoretical way this type of methods were mainly due to [18] while in a pratical way these methods were adapted by [13] to detect microembolic signals. The choice of the model is crutial to avoid false detections as it was the case in [13]. In that study the model used was based on the auto-regressive (AR) model. That model is known to be sentivitive both to the energy and frequency variations. Such a detector uses a constant threshold based on the prediction error for instance. Unfortunately, by using such model it was observed, for few patients, periodical false detections. Indeed, such periodical micro-events were detected when the blood flow passes from the diastolic to the systolic phase. This drawback was corrected by reducing the frequency sensitivity of the detector. A recent improvement of such detector was proposed in [19] by taking into account the heteroscedasticity [20] (specific case of non-stationarity where the variance is timevarying) of the random fluctuation of the prediction error. This detector name GARCH Model and derived from econometry domain was succefully applied to Doppler signals [19].

Notice that instead of basing the detector on rapid change model, it is also possible to take into account heteroskedastic and cyclostationary properties in the energy random fluctuations [21, 22]. In that case, the flucutation of the time-varying energy is obtained by removing the local trend (with a moving 

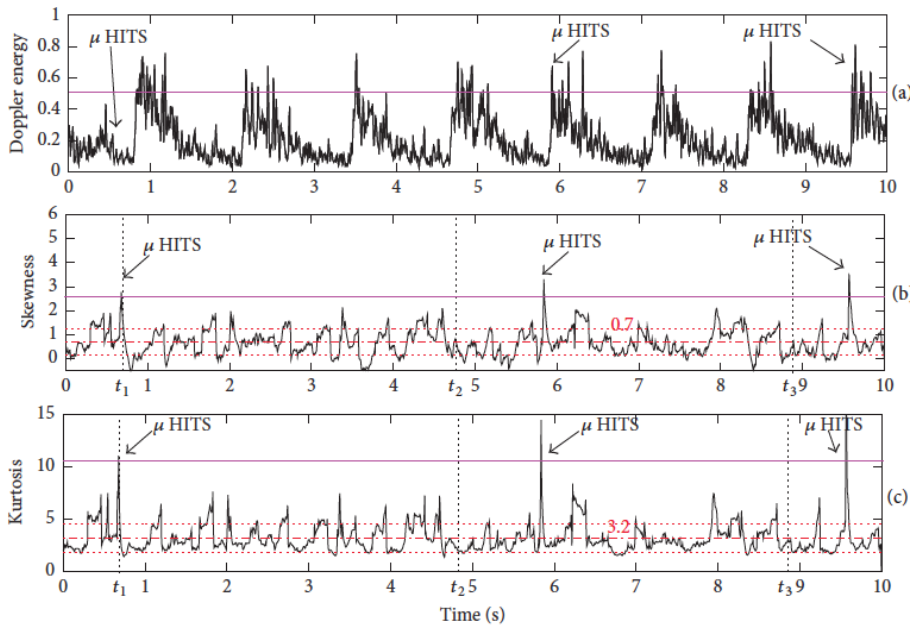

FIGURE 4 - Short term high order statistic (HOS) calculated from the Doppler signal. Time-varying energy (a), skewness (b) and kurtosis (c) calculated with a slidding window from the Doppler signal. Three high intensity transient signals (HITS) detected from HOS and two HITS detection from second order statistic (Energy).

average process) from the energy Doppler signal (see Figure 3 (a)). From the fluctuation energy, the envelop of the negative energy is calculated (see Figure 3 (b)) and serves as a threshold for the positive energy fluctuation (see Figure 3 (c)).

Another important property that is uncirconvent in the study of the blood Doppler signal is the cyclostationarity property (statistically stationary per cycle). A first attempt was done theoretically in [23] by considering the spectral correlation of the Doppler signal. Note that high order statistic (HOS) calculated from Doppler energy signal may be also use to detect microemboli [24]. Time varying energy and HOS are presented on Figure 4.

Another idea based on a synchronized detection with the cardiac rythm and the use of wavelet pack was proposed in [25]. Later a combination with an AR modeling and a synchronized detection was proposed in [26]. Another completely original approach was to combine an expert model with an AR model through a neurofuzzy approach [27]. Note that the first attempt to introduce an expert system was due to [28, 29]. One year after the introduction of the fuzzy-based method, an automated feature extraction and emboli detection system based on the principal component analysis and fuzzy sets was introduced in [30] .

Another interesting point was based on the radio-frequency (RF) signal instead of the Doppler signal. Initial works were done by [31, 32, 33], then lot of research teams performed a classification of high intensity transient signals (HITS). Machine learning approaches such as support vector machine, $\mathrm{k}$ nearest neighboors, neural network $[34,35]$ showed that it was possible to discriminate in vitro gaseous bubbles from solid micro-emboli. However these studies were not applied from clinical examinations.
The last but not least research works were based on the use of the 2D spectrogram as an image [36]. The different detectors proposed a $2 \mathrm{D}$ threshold for detecting time-frequency microembolic signatures. The main idea of these approaches was to mimic the human behavior during the manual human detection.

Will the advent of artifical intelligence revolutionize the emboli detection, only time will tell us?

\section{Références}

[1] J.L. Smith, D.H. Evans, L. Fan, A.J. Thrush, et A.R. Naylor. Processing doppler ultrasound signals from bloodborne emboli. Ultrasound Med. Biol., vol. 20, pp. 455-462, 1994.

[2] A. King, H. S. Markus, Doppler embolic signals in cerebrovascular disease and prediction of stroke risk, Stroke vol. 40, pp 3711-3717, 2009.

[3] E. Chung, L. Fan, C. Degg, D.H. Evans, Detection of Doppler embolic signals :psychoacoustic considerations, Ultrasound Med. Biol., vol. 31, pp. 1177-1184, 2005.

[4] J. Molloy and H. S. Markus, "Multigated doppler ultrasound in the detection of emboli in a flow model and embolic signals in patients," Stroke, vol. 27, pp. 1548-1552, 1996.

[5] R. Brucher and D. Russell, "Automatic embolus detection and artifact rejection with the first multifrequency transcranial doppler," Stroke, vol. 33, pp. 1969-1974, 2002.

[6] Moehring M.A., Spencer M.P., Power M-mode Doppler (PMD) for observing cerebral blood flow and tracking emboli, Ultrasound Med. Biol., vol. 28, pp. 49-57, 2002.

[7] A.D. Mackinnon, R. Aaslid, H.S. Markus, Long-term ambulatory monitoring forcerebral emboli using transcranial Doppler ultrasound, Stroke, vol. 35, pp. 73-78, 2004.

[8] E. Roy, P. Abraham, S. Montresor, and J. Saumet, "Comparaison of timefrequency estimators for peripheral embolus detection," Ultrasound Med. Biol., vol. 26, pp. 419423, 2000.

[9] C. Guetbi, D. Kouame, A. Ouahabi, and J. Remenieras, "New emboli detection methods," in Proc. IEEE Int. U1trason. Symp., vol. 2, pp. 1119-1122, 1997.

[10] P. Lui, B. Chan, F. Chan, P. Poon, H.Wang, and F. Lam, "Wavelet analysis of embolic heart sound detected by precordial doppler ultrasound during continuous venous air embolism in dogs," Anesth. Analg., vol. 2, pp. 325-331, 1998.

[11] N. Aydin, S. Padayachee, and H. Markus, "The use of the wavelet transform to describe embolic signals," Ultrasound Med. Biol., vol. 25, pp. 953-958, 1999.

[12] B. Krongold, A. Sayeed, M. Moehring, J. Ritcey, M. Spencer, and D. L. Jones, "Time-scale detection of microemboli in flowing blood with doppler ultrasound," 
IEEE Trans. Biomed. Eng., vol. 46, no. 9, pp. 1081-1089, Sep. 1999.

[13] J.-M. Girault, D. Kouame, A. Ouahabi, and F. Patat, "Micro-emboli detection : An ultrasound doppler signal processing viewpoint," IEEE Trans. Biomed. Eng., vol. 47, no. 11, pp. 1431-1438, Nov. 2000.

[14] J.-M. Girault, Cerebral microembolism synchronous detection with wavelet packets, in : Signal and Image Multiresolution Analysis, Wiley Online Library,2013, pp. 245287 (Chapter 4).

[15] G. Devuyst, P. Despland, and J. Bougousslavsky, "The matching pursuit : A new method of characterizing microembolic signal," Ultrasound Med. Biol., vol. 26, pp. 1051-1056, 2002.

[16] M. Geryes, S. Ménigot, J. Charara, J.-M. Girault, An energy detector based on negative energy fluctuations coupled with sub-band decomposition as a robust microembolus detection system, in : 2017 IEEE International Ultrasonics Symposium (IUS), IEEE, pp. 1-4, 2017.

[17] N. Aydin, F. Marvasti, H.S. Markus, Embolic Doppler ultrasound signal using discrete wavelet transform, IEEE Trans. Inf. Technol. Biomed., vol. 8, pp. 82-190, 2004.

[18] I.V. Nikiforov et I.N. Tikhonov. Detection of abrupt changes in signals and dynamical systems, Incis. IEEE Trans. Inform. Theory, vol. 77, pp. 355-373, 1986.

[19] J.-M. Girault, S. Ménigot, L. Deibine, Automatic detection of micro-emboli by means of a generalized autoregressive conditional heteroskedasticity model,J. Acoust. Soc. Am. vol. 128, pp 2304, 2010.

[20] H. White, A heteroskedasticity-consistence covariance matrix estimator and adirect test for heteroskedasticity, Econometrica, vol. 48, pp. 817-838, 1980.

[21] M. Geryes, S. Ménigot, W. Hassan, M. Almar, B. Guibert, C. Gautier, J. Charara,J.-M. Girault, A new energy detector of micro-emboli using a time-varyingthreshold, in : Third International Conference on Advances in BiomedicalEngineering (ICABME), IEEE, pp. 89-92, 2015.

[22] Maroun Geryes, Sébastien Ménigot, Jamal Charara, Mohammad Nasserdine,Ali Mcheick, Jean-Marc Girault, Enhanced weak Doppler micro-embolic signal detection using energy fluctuations, Biomedical Signal Processing and Control, vol. 47, pp. 177-182, 2019.

[23] Jean-Marc Girault, Mathieu Biard, Denis Kouamé, Aurore Bleuzen, and François Tranquart, "Spectral Correlation of the embolic blood Doppler signal", ICASSP, Toulouse, France, 2006.

[24] Maroun Geryes, Sebastien Ménigot, Walid Hassan, Ali Mcheick, Jamal Charara, and Jean-Marc Girault, Detection of Doppler Microembolic Signals Using High Order Statistics,Computational and Mathematical Methods in Medicine, Article ID 3243290, 8 pages, 2016.
[25] Jean-Marc Girault, Denis Kouamé, and François Tranquart, "Synchronous Detection of Emboli by wavelet packet decomposition", ICASSP, Honolulu, USA, pp. 409412, 2007.

[26] S. Ménigot, L. Dreibine, N. Meziati, J.-M. Girault, Automatic detection of microemboli by means of a synchronous linear prediction technique, in : 2009 IEEE International Ultrasonics Symposium (IUS), IEEE, pp. 23712374, 2009.

[27] Denis Kouamé, Mathieu Biard, Jean-Marc Girault, and Aurore Bleuzen, Adaptive AR and Neurofuzzy Approaches : Access to Cerebral Particle Signatures, IEEE Trans. on Information Technology in Biomedicine, vol. 10, pp. 559-566, 2006.

[28] V. Kemeny, D.W. Droste, S. Hermes, D. G. Nabavi, G. S.Altedorneburg, M. Siebler, and E. B. Ringelstein, "Automatic embolus detection compared with human experts : A doppler ultrasound study," Stroke, vol. 27, pp. 18401843, 1996.

[29] L. Fan, D. Evans, and A. Naylor, "Automated embolus identification using a rule-based expert system," Ultrasound Med. Biol, vol. 27, pp. 1065-1077, 2001.

[30] Da Xu, Yuanyuan Wang, An automated feature extraction and emboli detection system based on the PCA and fuzzy sets, Computers in Biology and Medicine, vol. 37, pp. 861-871, 2007.

[31] Mess WH, Willigers JM, Ledoux LA, Ackerstaff RG, Hoeks AP. Microembolic signal description : A reappraisal based on a customized digital postprocessing system. Ultrasound Med Biol, vol. 28, pp. 1447-1455, 2002.

[32] Cowe J, Gittins J, Naylor AR, Evans DH. RF signals provide additional information on embolic events recorded during TCD monitoring. Ultrasound Med Biol, vol. 31, pp. 613-623, 2005.

[33] Cowe J, Evans DH. Automatic detection of emboli in the TCD RF signal using principal component analysis. Ultrasound Med Biol, vol. 32, pp. 1853-1867, 2006.

[34] K. Ferroudji, N. Benoudjit, M. Bahaz, A. Bouakaz, Feature Selection Based on Rf Signals and Knn Rule : Application to Microemboli Classification, 7th International Workshop on Systems, Signal Processing and their Applications (WOSSPA), Algeria, pp. 251 - 254, 2011.

[35] N. Benoudjit, K. Ferroudji, M. Bahaz, A. Bouakaz, In vitro microemboli classification using neural network models and RF signals, Ultrasonics, vol. 51, pp. 247-252, 2011.

[36] B. K. Guepie, M. Martin, V. Lacrosaz, M. Almar, B. Guibert, P. Delachartre, Sequential emboli detection from ultrasound outpatient data, IEEE Journal of Biomedical and Health Informatics, vol. 99, pp. 334-341, 2018. 